\title{
ISOTOPIC AND ELEMENTAL COMPOSITION OF THE ANOMALOUS LOW-ENERGY COSMIC-RAY FLUXES
}

\author{
R. A. Mewaldt, E. C. Stone, S. B. Vidor, and R. E. Vogt \\ California Institute of Technology \\ Received 1975 August 20
}

\begin{abstract}
Recent observations of cosmic rays below $\sim 30 \mathrm{MeV}$ nucleon ${ }^{-1}$ have revealed anomalous enhancements in the energy spectra of oxygen, nitrogen, and helium relative to those of other nuclei such as boron and carbon. We have measured the quiet-time fluxes of the elements hydrogen through oxygen in the $\sim 4-30 \mathrm{MeV}$ nucleon ${ }^{-1}$ energy interval during the period 1972 October through 1974 October. We find that the low-energy fluxes of $\mathrm{Li}, \mathrm{Be}, \mathrm{B}$, and $\mathrm{C}$ are consistent with those expected from adiabatic deceleration and show no significant evidence for secondary fragmentation products arising from the enhanced nitrogen and oxygen fluxes. In the $\sim 6-12$ $\mathrm{MeV}$ nucleon ${ }^{-1}$ interval, the observed nitrogen and oxygen nuclei are predominantly ${ }^{14} \mathrm{~N}$ and ${ }^{16} \mathrm{O}$, with upper limits $\left(84 \%\right.$ confidence level) of ${ }^{15} \mathrm{~N} / \mathrm{N} \leq 0.26,{ }^{17} \mathrm{O} / \mathrm{O} \leq 0.13$, and ${ }^{18} \mathrm{O} / \mathrm{O} \leq 0.12$ for the other stable nitrogen and oxygen isotopes. These isotopic measurements are consistent with the presumed isotopic composition of the neutral interstellar medium, as would be the case if the anomalous component is locally accelerated. If, on the other hand, the enhanced low-energy cosmic rays result from unusual nucleosynthesis processes which enhance nitrogen and oxygen but not carbon, then these processes are constrained to producing predominantly ${ }^{14} \mathrm{~N}$ and ${ }^{16} \mathrm{O}$.
\end{abstract}

Subject heading: cosmic rays: abundances

\section{INTRODUCTION}

Recent observations of quiet-time low-energy cosmic rays have revealed anomalous enhancements of helium, nitrogen, and oxygen relative to other nuclei such as hydrogen, boron, and carbon (Garcia-Munoz, Mason, and Simpson 1973; Van Hollebeke, Wang, and McDonald 1973; Hovestadt et al. 1973; McDonald et al. 1974). For example, at $10 \mathrm{MeV}$ nucleon ${ }^{-1}$ the oxygen flux is enhanced by a factor of $\sim 30$ above that expected from an extrapolation of the spectrum of 40 to $100 \mathrm{MeV}$ nucleon ${ }^{-1}$ galactic cosmic rays. A similar enhancement is observed in the low-energy nitrogen, but not in the boron, carbon, magnesium, or silicon fluxes with energies down to $\sim 8 \mathrm{MeV}$ nucleon ${ }^{-1}$. This anomalous composition suggests that the origin of the enhanced fluxes is different from that of higher energy cosmic rays. Measurements of isotopic abundances may provide a discriminator between possible origins for these low-energy cosmic rays.

Using the Caltech Electron/Isotope Spectrometer (EIS) aboard the IMP-7 satellite, we have investigated the isotopic composition of $\sim 6-12 \mathrm{MeV}$ nucleon $^{-1}$ nitrogen and oxygen nuclei. In addition, we have used the EIS on both IMP-7 and IMP-8 to measure the fluxes of individual elements with $1 \leq Z \leq 8$ in the energy region of $\sim 4-30 \mathrm{MeV}$ nucleon ${ }^{-1}$, thus extending the search for possible enhancements of other elements to lower energies than previously reported. A preliminary account of this work has been given elsewhere (Hurford et al. 1974a; Stone et al. 1975; Mewaldt et al. 1975a,b).

\section{INSTRUMENTATION}

A schematic illustration of the IMP-7 EIS telescope is shown in Figure 1a. The telescope consists of a stack of fully depleted surface-barrier solid-state detectors surrounded by a plastic scintillator anticoincidence cup. For isotope analysis a $\Delta E$ versus $E$ technique is used. Particles are required to trigger the $50 \mu$ thick detector D2 and the $1 \mathrm{~mm}$ thick detector D5, with all other detectors in anticoincidence. In this analysis mode the annular detectors D0, D1, D3, and D4 are active collimators and provide a clean, low-background geometry. As a result of the collimation, the geometrical factor for D2D5 events is $0.07 \mathrm{~cm}^{2} \mathrm{sr}$. A more detailed discussion of the IMP-7 EIS can be found in the report by Hurford et al. (1974b).

The IMP-8 EIS is schematically illustrated in Figure $1 b$. The geometrical factor of $0.23 \mathrm{~cm}^{2} \mathrm{sr}$ for D2D5 events allows the elemental fluxes to be determined with better statistical precision than with the IMP-7 EIS. In order to obtain the fluxes at higher energies, a $\Delta E$ versus $E$ technique was used in one of two analysis modes. The narrow geometry mode, which was used to calculate the spectra of elements with $Z \geq 3$, includes particles that pass through the apertures of the annular detectors. For narrow geometry events that penetrate past D5 into D6 or further, the energy loss in D5 is obtained along with the residual energy loss in the remaining detectors. The hydrogen and helium spectra above $13 \mathrm{MeV}$ nucleon ${ }^{-1}$ were obtained from the wide geometry analysis mode, in 


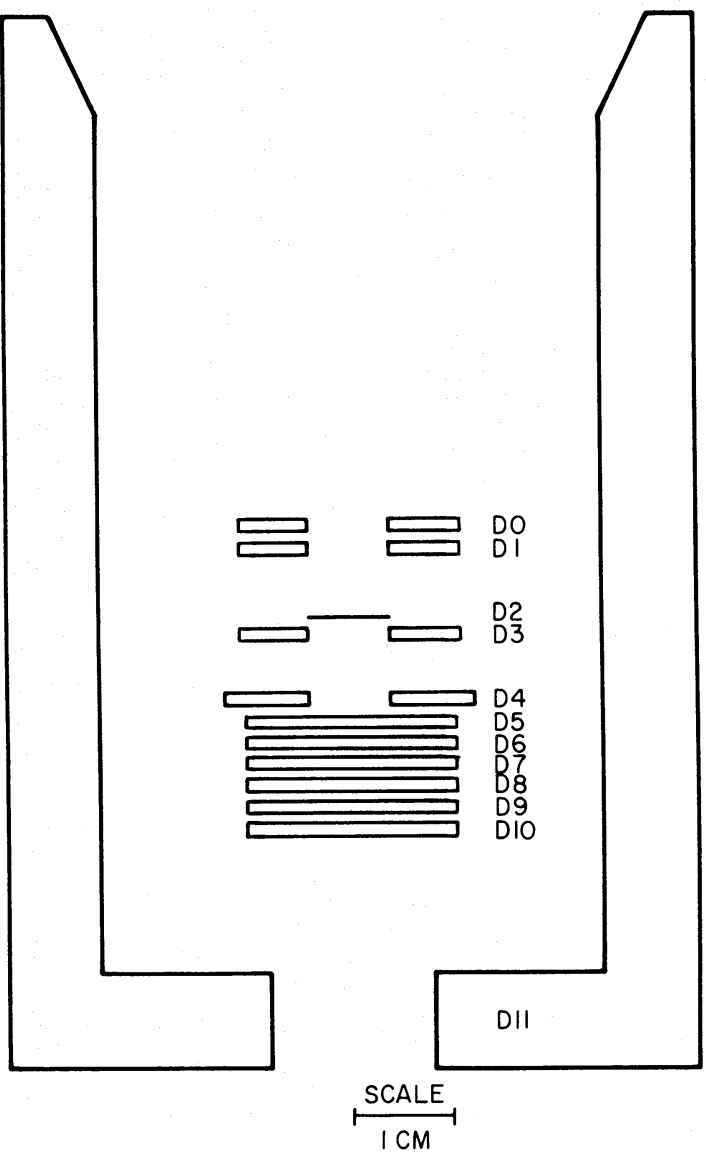

$a$

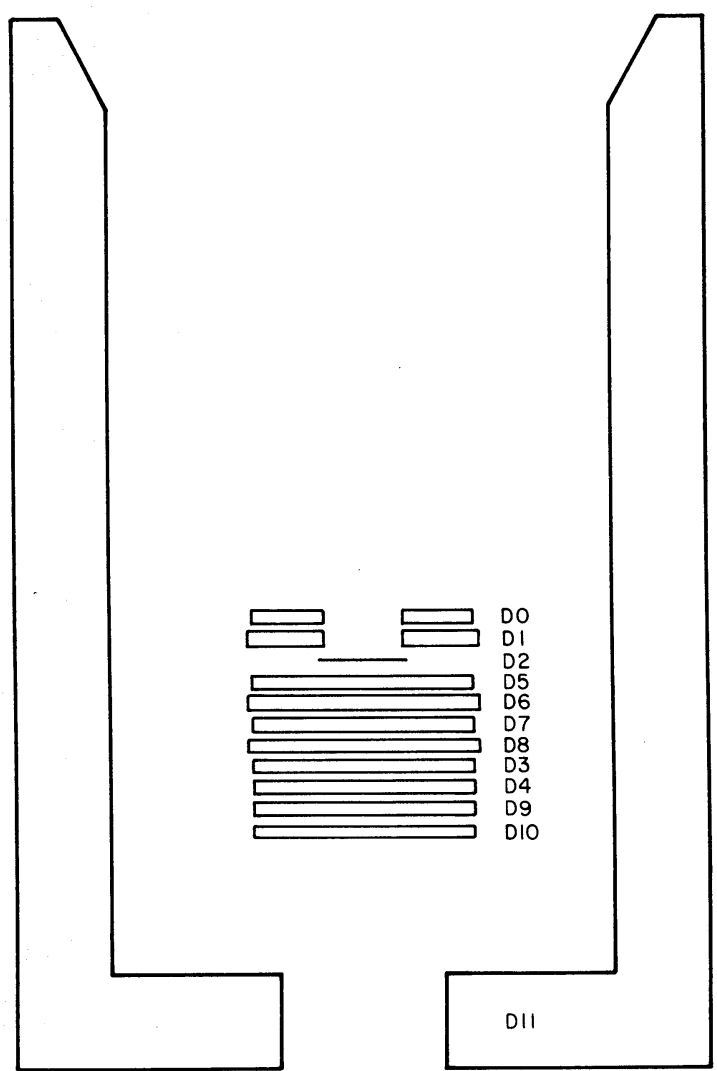

$b$

FIG. 1.-(a) Schematic illustration of the IMP-7 EIS. (b) Schematic illustration of the IMP-8 EIS.

which $\Delta E$ is measured in $\mathrm{D} 0$, and the residual energy is measured in D1 and D3 through D9.

\section{QUIET-TIME OBSERVATIONS}

To obtain solar quiet-time spectra of the low-energy cosmic rays, restrictions had to be placed on solar particle activity. We have used the IMP-8 EIS to measure the fluxes of elements with $3 \leq Z \leq 8$ during solar quiet periods, which we have defined as those days on which the average 4.4-12.9 MeV proton flux is $\leq 0.013\left(\mathrm{~cm}^{2} \mathrm{~s} \mathrm{sr}\right)^{-1}$. A comparable criterion was used for the IMP-7 EIS. This definition is a useful one since the combined flux of low-energy carbon, nitrogen, and oxygen was found to be essentially independent of the 4.4-12.9 MeV proton flux during these solar quiet periods and is not sensitive to the exact value of the limit placed on the proton flux. Days on which the average 4.4-12.9 MeV proton flux is greater than $0.013\left(\mathrm{~cm}^{2} \mathrm{~s} \mathrm{sr}\right)^{-1}$ are referred to as solar active periods. For hydrogen and helium, the fluxes were obtained from the IMP-7 EIS for a period of 35 nonconsecutive days during which the $\sim 2 \mathrm{MeV}$ proton flux was near the lowest level during the period 1972 October to 1974 September.
A plot of $\Delta E$ versus residual energy for all our IMP-7 D2D5 data with $Z>2$ is shown in Figure $2 a$ for the time period 1972 October to 1974 October. For clarity, events that saturate the analog-to-digital converter of either D2 or D5 (i.e., events along the top or righthand border of the plot) are not shown. The solid curves indicate the predicted tracks for the stable isotopes of $\mathrm{Li}$ through $\mathrm{O}$ based on an accelerator calibration of a spare telescope. As a result of the calibration, these tracks are known to within $\sim 0.05 \mathrm{amu}$ (Vidor 1975).

Figure $2 b$ shows a plot of $\Delta E$ versus residual energy for all our solar quiet-time IMP-8 D2D5 data with $Z>3$ for the time period 1973 November to 1974 September. The white bands of $5 \mathrm{amu}$ width are used to identify elements. The rms mass resolution of the elements within the bands agrees with the resolution predicted from the physical parameters of the detector system (except for beryllium, where some background is evident). Since the rms mass resolution is $\sim 1 \mathrm{amu}$ for oxygen and is correspondingly smaller for the lighter elements, the 5 amu-wide bands of Figure $2 b$ include essentially all real particles. The better mass resolution makes the IMP-7 EIS more appropriate for measuring the isotopic composition, while the larger 


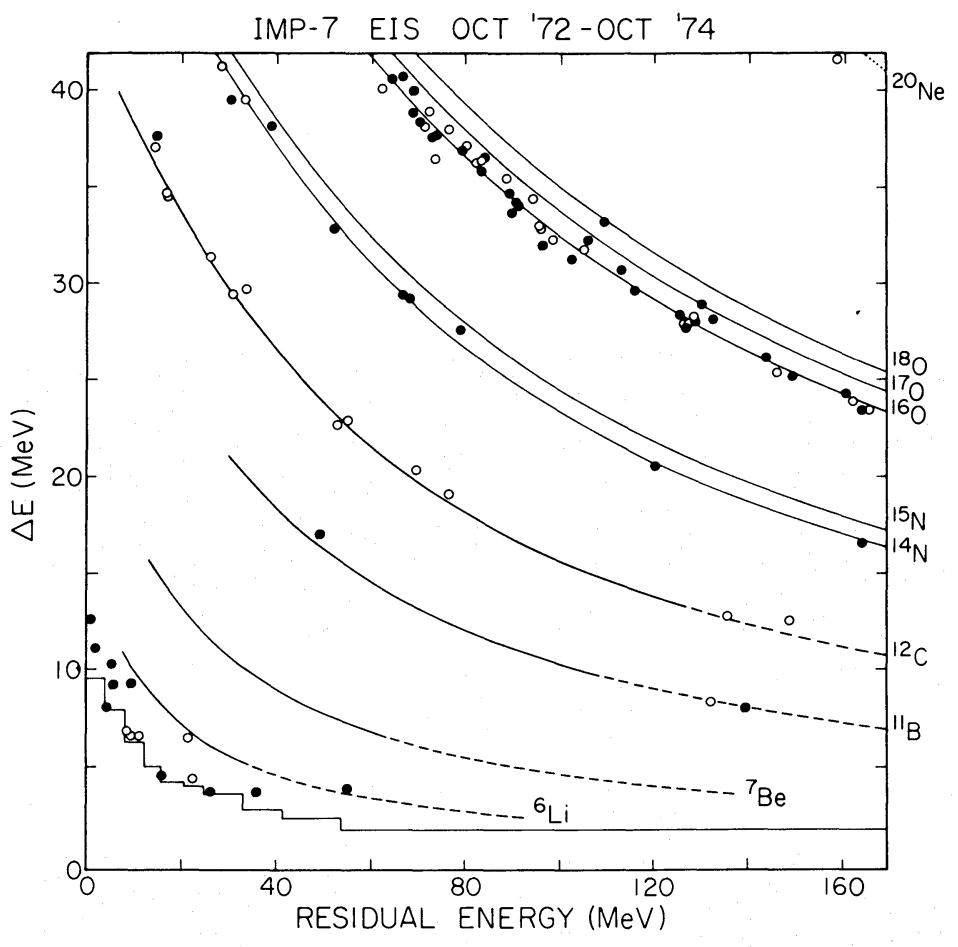

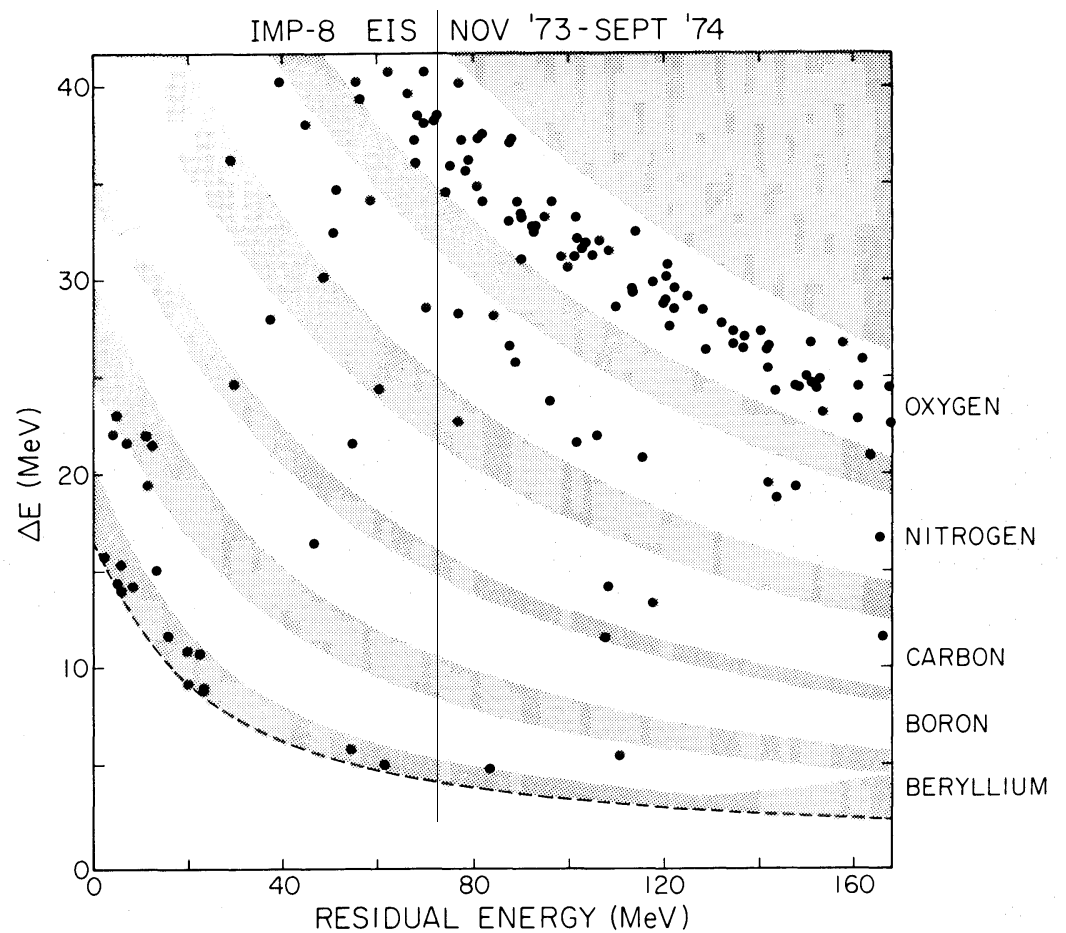

FIG. 2.-(a) Plot of $\Delta E$ versus residual for D2D5 events with $Z>2$ : $९$, solar quiet data; $O$, solar active data. Solid curves are predicted isotope tracks based on accelerator calibrations. Dashed curves are extrapolated from calibration data. Dotted curve for ${ }^{20} \mathrm{Ne}$ is based on calculation only. No data are shown below the broken line. (b) $\Delta E$ versus residual energy plot of solar quiet time 1MP-8 D2D5 data from 1973 October 31 to 1974 September 25 . No data below the dashed line are included in the plot. High-energy beryllium particles can penetrate past D5 into D6 before saturating the D5 ADC, giving rise to the cutoff of the beryllium 
geometrical factor makes the IMP-8 EIS appropriate for calculating the $Z>3$ elemental fluxes.

\section{ELEMENTAL SPECTRA}

Quiet-time spectra for the elements $\mathrm{H}, \mathrm{He}, \mathrm{C}, \mathrm{N}$, and $\mathrm{O}$ are shown in Figure 3. Also shown are higher energy spectra of other experimenters. The $\mathrm{H}$ and $\mathrm{He}$ spectra below $\sim 5-8 \mathrm{MeV}$ nucleon $^{-1}$ exceed the quietest spectra observed (see Mewaldt, Stone, and Vogt 1975c) and are therefore very likely of solar origin. In the energy region $\sim 10-40 \mathrm{MeV}$ nucleon ${ }^{-1}$, the hydrogen and carbon fluxes are consistent with a $j \propto T$ relation (Rygg and Earl 1971), where $j$ is the differential flux and $T$ is the kinetic energy per nucleon. This simple linear relationship can be explained with conventional modulation theory by the adiabatic deceleration of galactic cosmic rays in the heliosphere (Goldstein, Ramaty, and Fisk 1970). By contrast, the nitrogen and oxygen fluxes increase rapidly at energies below $30 \mathrm{MeV}$ nucleon ${ }^{-1}$. The helium flux is relatively energy independent from 5 to $40 \mathrm{MeV}$ nucleon ${ }^{-1}$. In this respect the helium flux is also anomalous since, at low energies, it is significantly above a $j \propto T$ extrapolation. At energies of overlap, the present observations are consistent with the 1972-1973 observations of other experimenters (Garcia-Munoz, Mason, and Simpson 1973; Van Hollebeke, Wang, and McDonald 1973; Hovestadt et al. 1973; McDonald et al. 1974; Chan and Price 1974).

In Figure 4 the observed fluxes of elements with $3 \leq Z \leq 7$ are compared with two spectral types:

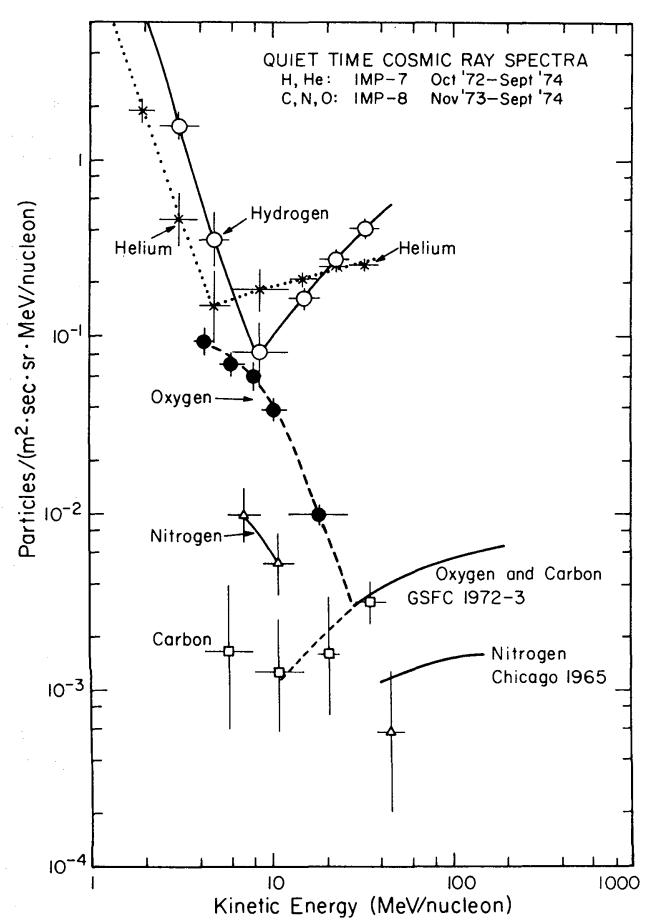

FIG. 3.-Quiet-time spectra. The smooth curves are meant to aid the eye and are not best fits to the data.

(1) spectra with an energy dependence identical to that of the 4-60 MeV nucleon ${ }^{-1}$ oxygen spectrum shown in Figure 3 but normalized by the high energy

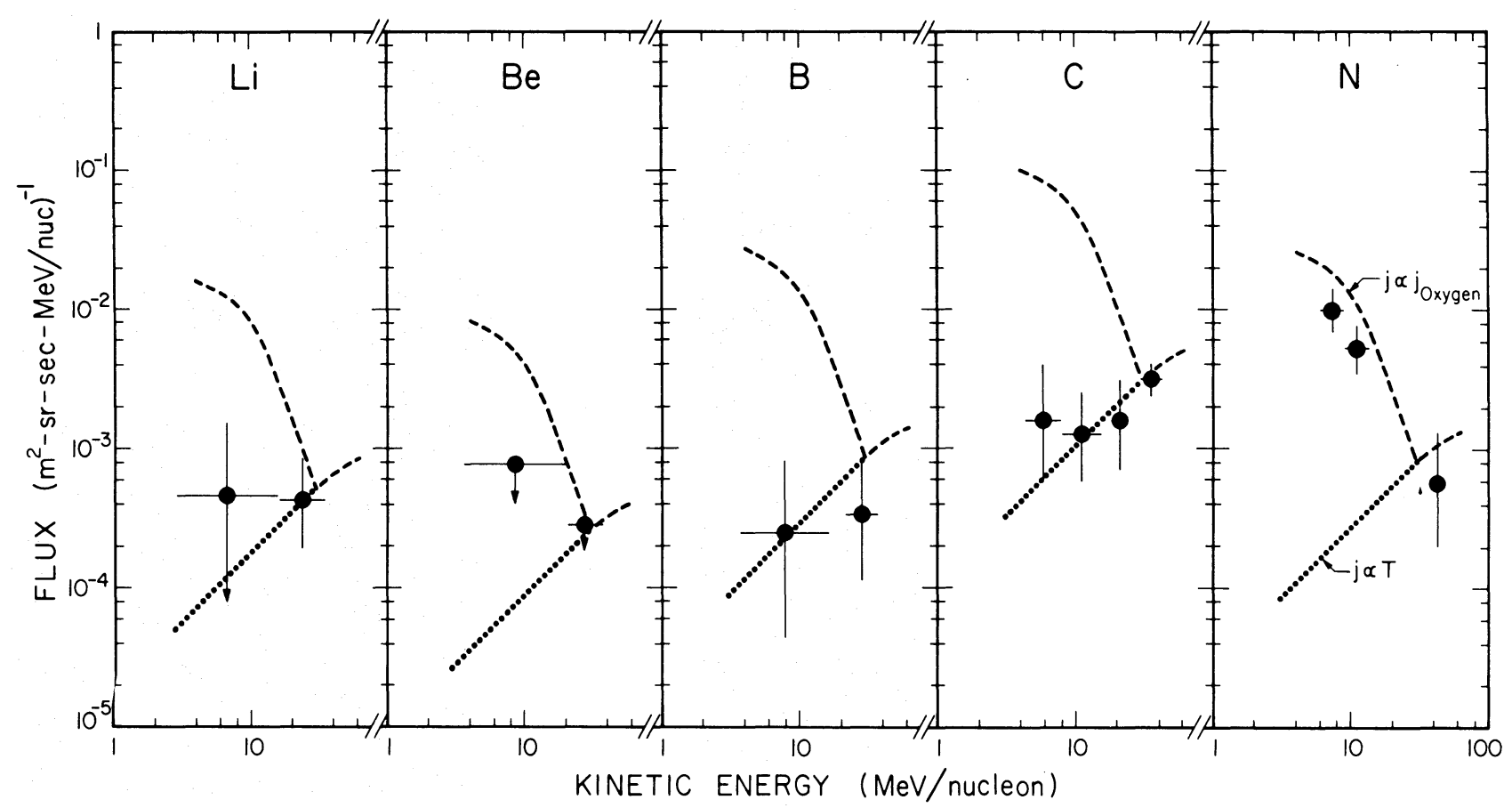

Fis. 4.-Quiet-time fluxes from 1972 October to 1974 September. The curves are normalized at $30 \mathrm{MeV}$ per nucleon to the highenergy cosmic-ray abundances relative to oxygen. The shape of the $j_{\text {oxygen }}$ curve is from Fig. 3. The lowest energy Li point is obtained from the IMP-7 EIS. All other points are obtained from the IMP-8 EIS. 


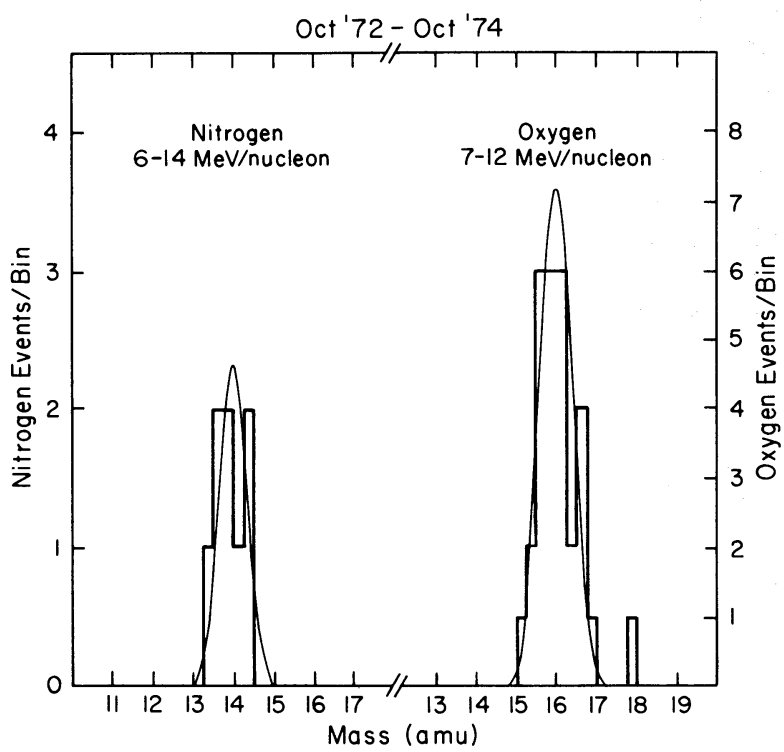

FIG. 5.-Mass histograms of quiet time nitrogen and oxygen data. The Gaussian curves show the predicted instrument response to ${ }^{14} \mathrm{~N}$ and ${ }^{16} \mathrm{O}$ cosmic rays.

abundance of each element relative to oxygen (Webber, Damle, and Kish 1972) and (2) spectra of the form $j \propto T$ below $30 \mathrm{MeV}$ nucleon ${ }^{-1}$. We find that even at the lowest energies only the nitrogen flux exhibits an enhancement similar to oxygen. There is no similar enhancement of the $3 \leq Z \leq 6$ fluxes, which are consistent with $j \propto T$, although, because of limited statistics, fluxes that are independent of energy cannot be ruled out.

\section{ISOTOPIC COMPOSITION}

A nonintegral effective mass can be assigned to each data point in Figure $2 a$ by properly interpolating between the integral mass tracks. The resulting mass histograms of the solar quiet-time nitrogen and oxygen data are shown in Figure 5. The Gaussian curves superposed on the histograms indicate the expected response to a pure ${ }^{14} \mathrm{~N}$ and ${ }^{16} \mathrm{O}$ composition. The widths of the curves represent the predicted mass resolution and are calculated from the physical parameters of the detector system. From the accelerator calibration it was determined that the technique used for obtaining the predicted mass resolution is accurate to better than 10 percent (Vidor 1975).

Excluding the oxygen event near mass 18 , the observed mean mass and the rms mass resolution are compared in Table 1 with the values expected for a pure ${ }^{14} \mathrm{~N}$ and ${ }^{16} \mathrm{O}$ composition. The mean mass and the mass resolution of the solar quiet-time nitrogen and oxygen data are consistent with the values expected from a pure ${ }^{14} \mathrm{~N}$ and ${ }^{16} \mathrm{O}$ composition and are inconsistent with a large fraction of either ${ }^{15} \mathrm{~N}$ or of ${ }^{17} \mathrm{O}$ or ${ }^{18} \mathrm{O}$, which are the other stable isotopes of nitrogen and oxygen. As a consistency check on our knowledge of the mass response to nitrogen and oxygen, we have calculated the observed mean mass and rms mass resolution of the nitrogen and oxygen events obtained during solar active periods. The observed dominance of ${ }^{14} \mathrm{~N}$ and ${ }^{16} \mathrm{O}$ during solar active periods is expected if the solar system abundances of Cameron (1973) are representative of solar cosmic rays. It should be noted that the expected mass resolution listed in Table 1 for the solar quiet nitrogen differs from the mass resolution expected for the solar active nitrogen because the average energies of the two sets of data differ and because the mass resolution of the instrument is somewhat energy dependent.

Including the oxygen event near mass 18 , upper limits to the abundances of ${ }^{15} \mathrm{~N},{ }^{17} \mathrm{O}$, and ${ }^{18} \mathrm{O}$ in the low-energy solar quiet-time nitrogen and oxygen cosmic rays were obtained by means of the maximum likelihood technique. The results are given in Table 2 . The limits obtained in Table 2 are not sensitive to the assumed mass resolution of the instrument, although they do depend somewhat on our knowledge of the mass response. A shift of $0.05 \mathrm{amu}$ in the location of the nominal ${ }^{14} \mathrm{~N}$ and ${ }^{16} \mathrm{O}$ response curves would change the resulting ${ }^{15} \mathrm{~N} / \mathrm{N}$ and ${ }^{17} \mathrm{O} / \mathrm{O}$ upper limits by 0.03 and the ${ }^{18} \mathrm{O} / \mathrm{O}$ upper limit by 0.01 .

\section{DISCUSSION}

The present results, which extend earlier observations to lower energies, confirm the anomalous characteristics of the elemental abundances over the entire 4-30 MeV nucleon ${ }^{-1}$ energy interval. The elemental composition at $\sim 5-12.5 \mathrm{MeV}$ nucleon $^{-1}$ relative to oxygen is summarized in Figure 6 . The

TABLE 1

Mass Distributions of Low-Energy Nitrogen and Oxygen Cosmic Rays

\begin{tabular}{|c|c|c|c|c|}
\hline \multirow[b]{2}{*}{ ELEMENT } & \multicolumn{2}{|c|}{ Solar Quiet Periods } & \multicolumn{2}{|c|}{ Solar Active Periods } \\
\hline & Observed & Expected* & Observed & Expected $*$ \\
\hline \multicolumn{5}{|l|}{ Nitrogen: } \\
\hline $\bar{M}(\mathrm{amu})$ & $13.94 \pm 0.13$ & 14.00 & $13.94 \pm 0.08$ & 14.00 \\
\hline$\sigma_{M}(\mathrm{amu}) \ldots \ldots \ldots \ldots$ & $0.38 \pm 0.10$ & 0.37 & $0.12(+0.25,-0.06)$ & 0.41 \\
\hline \multicolumn{5}{|l|}{ Oxygen: } \\
\hline $\bar{M}(\mathrm{amu})$. & $15.98 \pm 0.08$ & 15.99 & $15.92 \pm 0.10$ & 15.99 \\
\hline$\sigma_{M}(\mathrm{amu}) \ldots \ldots \ldots \ldots$ & $0.43 \pm 0.06$ & 0.42 & $0.46 \pm 0.07$ & 0.42 \\
\hline
\end{tabular}

* Calculated for pure ${ }^{14} \mathrm{~N}$ and ${ }^{16} \mathrm{O}$ composition. 
TABLE 2

Isotopic CoMposition of THE LOW-ENERgY NITROGEN AND OXYGEN COSMIC RAYS

\begin{tabular}{|c|c|c|}
\hline Isotope Fraction & Energy (MeV per nucleon) & $84 \%$ Confidence Interval \\
\hline $\begin{array}{l}{ }^{15} \mathrm{~N} / \mathrm{N} \ldots \ldots \ldots \ldots \ldots \ldots \\
{ }^{17} \mathrm{O} / \mathrm{O} \ldots \ldots \ldots \ldots \ldots \ldots \\
{ }^{18} \mathrm{O} / \mathrm{O} \ldots \ldots \ldots \ldots \ldots\end{array}$ & $\begin{array}{l}5.6-12.7 \\
7.0-11.8 \\
7.0-11.2\end{array}$ & $\begin{array}{l}\leq 0.26 \\
\leq 0.13 \\
\leq 0.12\end{array}$ \\
\hline
\end{tabular}

comparison of the composition of the low-energy fluxes with that of higher energy galactic cosmic rays (Webber, Damle, and Kish 1972; Shapiro, Silberberg, and Tsao 1973; Webber and Lezniak 1974) shown in Figure 6 illustrates the marked enhancement of nitrogen and oxygen. For example, at $\sim 5-12.5 \mathrm{MeV}^{\text {nucleon }}{ }^{-1}$ the relative abundances are $\mathrm{O} / \mathrm{He} \sim 0.25, \mathrm{O} / \mathrm{B} \sim 100$, and $\mathrm{O} / \mathrm{C} \sim 30$. These ratios are strongly energy dependent, as can be determined from the spectra in Figures 3 and 4.

The present measurement, which indicates that ${ }^{14} \mathrm{~N}$ is the dominant nitrogen isotope in the low-energy cosmic rays, contrasts with results in the energy range of $\sim 50-250 \mathrm{MeV}$ nucleon $^{-1}$, which indicate that ${ }^{15} \mathrm{~N}$ comprises more than 50 percent of the nitrogen in these higher energy cosmic rays (Webber et al. 1973; Garcia-Munoz, Mason, and Simpson 1974). The ${ }^{15} \mathrm{~N}$ in the higher energy galactic cosmic rays is believed to result primarily from the fragmentation of oxygen cosmic-ray nuclei which have passed through approximately $6 \mathrm{gcm}^{-2}$ of interstellar material (Shapiro, Silberberg, and Tsao 1973). The relative lack of ${ }^{15} \mathrm{~N}$ in the low-energy cosmic rays is consistent

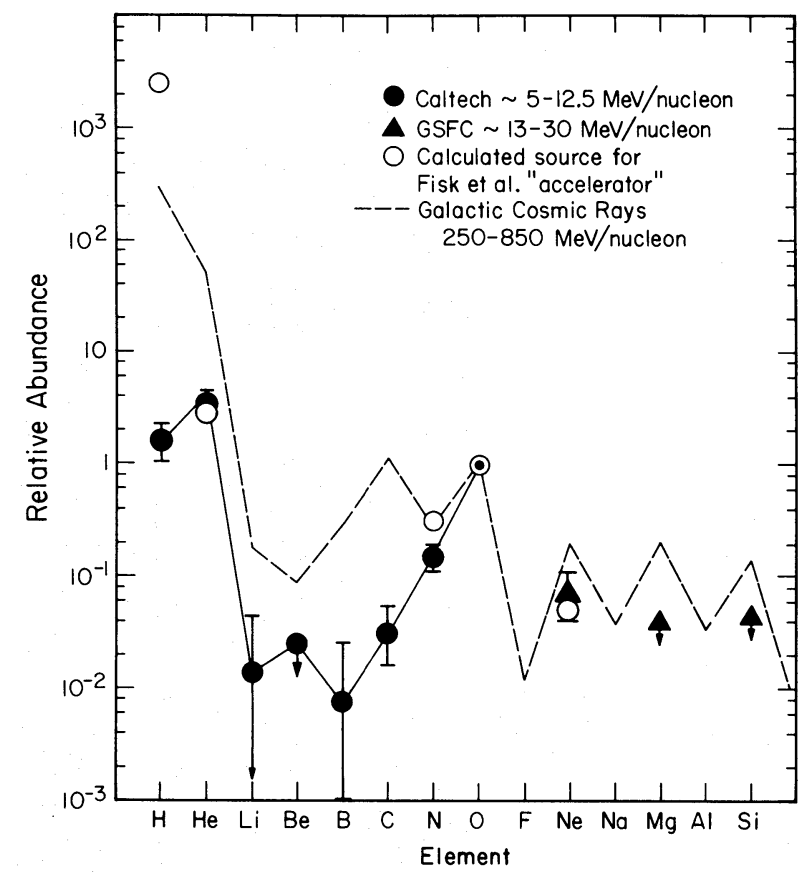

FIG. 6.-Comparison of observed abundances with the abundances of higher energy cosmic rays and the "source" abundances associated with the model of Fisk et al. (1974). The 13-30 MeV per nucleon observations are from McDonald et al. (1974) with the lack of secondary carbon, lithium, beryllium, and boron. Thus, the quiet-time cosmic rays observed at $\sim 10 \mathrm{MeV}$ nucleon ${ }^{-1}$ differ in both elemental and isotopic composition from the galactic cosmic-ray component observed at higher energies, providing strong evidence that the low-energy nitrogen and oxygen fluxes are not simply a sample of the higher energy component that has been decelerated in the heliosphere.

The lack of the secondary cosmic-ray ${ }^{15} \mathrm{~N}, \mathrm{Li}, \mathrm{Be}, \mathrm{B}$, and $\mathrm{C}$ indicates that the low-energy cosmic rays are not as contaminated with fragmentation products as are the higher energy cosmic rays. The low-energy cosmic rays observed near Earth therefore represent a relatively pure sample of the source material. If we assume that the abundances of the secondary ${ }^{15} \mathrm{~N}$, ${ }^{17} \mathrm{O}$, and ${ }^{18} \mathrm{O}$ relative to $\mathrm{Li}, \mathrm{Be}$, and $\mathrm{B}$ are the same at low energies as at high energies, then based on our observed low energy $\mathrm{Li}, \mathrm{Be}$, and $\mathrm{B}$ fluxes, we expect that secondary production would result in ratios of ${ }^{15} \mathrm{~N} / \mathrm{N} \sim 0.05,{ }^{17} \mathrm{O} / \mathrm{O} \sim 0.001$, and ${ }^{18} \mathrm{O} / \mathrm{O} \sim 0.001$. Thus, a finite amount of ${ }^{15} \mathrm{~N},{ }^{17} \mathrm{O}$, or ${ }^{18} \mathrm{O}$ that is somewhat below our measured upper limits would be indicative of the source composition.

Consideration of the elemental and isotopic composition of the low-energy cosmic rays leads to the identification of at least two distinct particle populations at low energies. The low-energy spectra of $\mathrm{H}$, $\mathrm{Li}, \mathrm{Be}, \mathrm{B}$, and $\mathrm{C}$ are consistent with $j \propto T$, as would result from adiabatic deceleration of higher energy galactic cosmic rays in the solar wind. The low-energy $\mathrm{He}, \mathrm{N}$, and $\mathrm{O}$ fluxes, however, may be part of an additional low-energy component which has a composition markedly different from that of higher energy cosmic rays.

It is interesting to note several similarities between the enhanced helium and the enhanced nitrogen and oxygen fluxes. From isotopic analysis, the spectrum of the secondary component ${ }^{3} \mathrm{He}$ is consistent with a $j \propto T$ relationship (Teegarden et al. 1975; GarciaMunoz, Mason, and Simpson 1975; Mewaldt, Stone, and Vogt $1975 d$ ). Only the isotope ${ }^{4} \mathrm{He}$ exhibits a relative enhancement, resulting in an enhanced ${ }^{4} \mathrm{He} /$ ${ }^{3} \mathrm{He}$ ratio at low energies. Thus, the isotopic compositions of nitrogen and helium show a similar energy dependence. In addition, the low-energy helium flux exhibits time variations similar to the low-energy nitrogen plus oxygen flux (Mewaldt, Stone, and Vogt 1975e).

Two possible sources of the anomalous low-energy cosmic rays have been proposed. Fisk, Kozlovsky, and Ramaty (1974) have suggested that the anomalous component originates from neutral interstellat particles 
that penetrate the heliosphere, become singly ionized, and are then accelerated to several $\mathrm{MeV}$ per nucleon. According to this model only those elements which are abundant in the neutral state in the interstellar medium (e.g., $\mathrm{H}, \mathrm{He}, \mathrm{N}, \mathrm{O}, \mathrm{Ne}$ ) and penetrate the heliosphere can be accelerated. Figure 6 compares the relative abundances of the low-energy cosmic rays with the estimated source abundances for the proposed accelerator. To estimate these source abundances, the interstellar abundances, adopted from the solar system abundances (Cameron 1973), were multiplied by the areas corresponding to the approximate radial distances from the Sun at which the different elements are ionized (Axford 1972). Only those elements which exist in the interstellar medium primarily in the neutral state (Rogerson et al. 1973) were considered, and no correction was made for the partial ionization of some of these elements.

Notice that there is qualitative agreement between the observed helium, nitrogen, oxygen, and neon fluxes and the relative source composition. However, the observed hydrogen flux is $\sim 10^{3}$ times lower than the source hydrogen, perhaps because of preferential acceleration of heavier nuclei or solar modulation effects. In order to make a more quantitative comparison of the observed fluxes with the fluxes expected from the model of Fisk, Kozlovsky, and Ramaty (1974), these effects would have to be considered. If there is no preferential acceleration of one isotope relative to another, then the model of Fisk et al. would result in an isotopic composition of the lowenergy cosmic rays similar to that of the neutral interstellar gas. If the solar system abundances (Cameron 1973) apply to the interstellar gas, then the locally accelerated low-energy nitrogen and oxygen cosmic rays are expected to be respectively 99.6 percent ${ }^{14} \mathrm{~N}$ and 99.8 percent ${ }^{16} \mathrm{O}$. The local-acceleration theory is therefore consistent with our observations that ${ }^{14} \mathrm{~N}$ and ${ }^{16} \mathrm{O}$ are the dominant isotopes of the low-energy nitrogen and oxygen cosmic rays.

If, on the other hand, the low-energy nitrogen and oxygen cosmic rays originate from a nearby galactic source, then any proposed nucleosynthesis process which is designed to produce enhancements of nitrogen and oxygen and depletion of carbon must simultaneously produce isotopic abundances consistent with our observations. The hot CNO cycle (Audouze, Truran, and Zimmerman 1973) or nova outbursts in white dwarfs (Starrfield et al. 1972; Hoyle and Clayton 1974) can produce an overabundance of nitrogen and oxygen relative to carbon. In particular, Hoyle and Clayton (1974) proposed that white-dwarf novae could be the source of the low-energy nitrogen and oxygen cosmic rays. They suggested that ${ }^{15} \mathrm{~N}$ and ${ }^{17} \mathrm{O}$ should be overabundant and, depending on initial conditions, perhaps even the dominant nitrogen and oxygen isotopes. The present measurements rule out any astrophysical source of these particles in which ${ }^{15} \mathrm{~N}$ and ${ }^{17} \mathrm{O}$ are more abundant than ${ }^{14} \mathrm{~N}$ and ${ }^{16} \mathrm{O}$.

Mr. W. E. Althouse, Dr. G. J. Hurford, and Dr. J. E. Lupton made significant contributions to the design and development of the Electron/Isotope Spectrometer. In addition, Dr. Hurford was responsible for many of the data analysis programs. This work was supported in part by the National Aeronautics and Space Administration under contract NAS5-11066 and grant NGR 05-002-160.

\section{REFERENCES}

Audouze, J., Truran, J. W., and Zimmerman, B. A. 1973, Ap.J., 184, 493.

Axford, W. I., 1972, in Solar Wind, ed. C. P. Sonnett, P. J. Coleman, Jr., J. M. Wilcox, NASA SP-308 (Washington: National Aeronautics and Space Administration), p. 609.

Cameron, A. G. W. 1973, in Explosive Nucleosynthesis, ed. D. N. Schramm and W. D. Arnett (Austin: University of Texas Press), p. 3

Chan, J. H., and Price, P. B. 1974, Ap. J. (Letters), 190, L39.

Fisk, L. A., Kozlovsky, B., and Ramaty, R. 1974, Ap. J. (Letters), 190, L35.

Garcia-Munoz, M., Mason, G. M., and Simpson, J. A. 1973, Ap. J. (Letters), 182, L81.

1974, talk presented at the Symposium on Measurements and Interpretation of the Isotopic Composition of Solar and Galactic Cosmic Rays, Durham, New Hampshire.

. 1975, Ap. J., 202, 265.

Goldstein, M. L., Ramaty, R., and Fisk, L. A. 1970, Phys. Rev. Letters, $25,832$.

Hovestadt, D., Vollmer, O., Gloeckler, G., and Fan, C. Y. 1973, Phys. Rev. Letters, 31, 650.

Hoyle, F., and Clayton, D. D. 1974, Ap. J., 191, 705.

Hurford, G. J., Mewaldt, R. A., Stone, E. C., Vidor, S. B., and Vogt, R. E. 1974a, Bull. Am. Phys. Soc., 19, 433.

Hurford, G. J., Mewaldt, R. A., Stone, E. C., and Vogt, R. E. $1974 b, A p . J ., 192,541$.

McDonald, F. B., Teegarden, B. J., Trainor, J. H., and Webber, W. R. 1974, Ap. J. (Letters), 187, L105.

Mewaldt, R. A., Stone, E. C., Vidor, S. B., and Vogt, R. E.

1975a, Proc. 14th International Cosmic Ray Conf., Munich, Paper OG 7-10. 1975b, ibid., Paper OG 10-10.

Mewaldt, R. A., Stone, E. C., and Vogt, R. E. 1975c, Proc 14th International Cosmic Ray Conf., Munich, Paper OG $10-6$.

1975d, ibid., Paper OG 7-2. 1975e, ibid., Paper OG 10-11.

Rogerson, J. B., York, D. G., Drake, J. F., Jenkins, E. B., Morton, D. C., and Spitzer, L. 1973, Ap. J. (Letters), 181, L110.

Rygg, T. A., and Earl, J. A. 1971, J. Geophys. Res., 76, 7445.

Shapiro, M. M., Silberberg, R., and Tsao, C. H. 1973, 13th International Cosmic Ray Conf., Denver, Conf. Papers, 1, 578.

Starrfield, S., Truran, J. W., Sparks, W. M., and Kutter, G. S. 1972, Ap. J., 176, 169.

Stone, E. C., Mewaldt, R. A., Vidor, S. B., and Vogt, R. E. 1975, Bull. Am. Phys. Soc., 20, 633.

Teegarden, B. J., von Rosenvinge, T. T., McDonald, F. B., Trainor, J. H., and Webber, W. R. 1975, Ap. J., 202, 815.

Van Hollebeke, M. A. I., Wang, J. R., and McDonald, F. B. 1973, 13th International Cosmic Ray Conf., Denver, Conf. Papers, 2, 1298.

Vidor, S. B. 1975, Ph.D. thesis, California Institute of Technology (unpublished).

Webber, W. R., Damle, S. V., and Kish, J. 1972, Ap. and Space Sci., 15, 245.

Webber, W. R., and Lezniak, J. A. 1974, Ap. and Space Sci., 30, 361 .

Webber, W. R., Lezniak, J. A., Kish, J., and Damle, S. V. 1973, Ap. and Space Sci., 24, 17.

R. A. Mewaldt, E. C. Stone, S. B. Vidor, and R. E. Vogt: California Institute of Technology, Mail Code 220-47, Pasadena, CA 91125 